\title{
Effects of the Type and Point of Inclusion of Soy-Melon-Protein Supplement on the Sensory Qualities of "Gari" Semolina
}

\author{
M. O. Oluwamukomi ${ }^{1}$ \\ ${ }^{1}$ Dept. of Food Science and Tech., Federal University of Technology, Akure, Nigeria \\ Correspondence: M. O. Oluwamukomi, Dept. of Food Science and Tech., Federal University of Technology, \\ P.M.B 704, Akure, Nigeria. Tel: 234-806-013-7692. E-mail: mukomi2003@yahoo.com
}

Received: February 27, 2012 Accepted: February 28, 2013 Online Published: March 27, 2013

doi:10.5539/jfr.v2n2p120 URL: http://dx.doi.org/10.5539/jfr.v2n2p120

\begin{abstract}
The effects of the type of enrichment and the stage of application of supplement on the sensory qualities of Soy-melon protein-enriched gari semolina were studied. Three protein supplements (Full fat, Defatted and Milk residue) were added to the gari meal before fermentation, after fermentation and after toasting. After toasting and cooling, the samples were subjected to sensory evaluation at weekly interval over a period of 32 weeks by 20 member sensory panel of the Federal University of Technology, Akure, Nigeria in order to determine its shelf life. The panel members were students and staff members who were used to the consumption and sensory evaluation of gari. They were instructed to evaluate differences in overall sensory quality between the control and other samples packaged in HDPE film and Woven sack and subjected to storage at 20,30 and $40^{\circ} \mathrm{C}$. A nine-point hedonic scoring system was used for the evaluation, where $1=$ extremely disliked and $9=$ extremely liked. Results obtained from other data on the flavor difference scores for each product were subjected to regression analysis based on the critical minimum panel mean score of 5.0 for shelf stability of the samples. The shelf lives for the samples at different storage conditions were determined from the slopes of the regression equations. Results showed that enrichment with soy-melon flour reduced the shelf life at a high temperature above $40^{\circ} \mathrm{C}$ from 148 weeks to 17 weeks. The shelf lives of samples packaged in HDPE were significantly higher than those packaged in woven sack. The shelf life was reduced significantly by increase in temperature which exhibited a negative correlation with the flavor scores.
\end{abstract}

Keywords: soy-melon-protein, 'gari', enrichment, sensory evaluation, shelf life

\section{Introduction}

Gari is a fermented, dewatered and toasted granules from cassava which is widely consumed all over West Africa and in Brazil where it is known as 'farinha de manioca' (Lancaster et al., 1982). Gari is one of the most popular forms in which cassava (Manihot utilisima) also known as manioc is consumed in Nigeria and some other parts of West Africa (Kordylas, 1990). It is a major component of everyday diet in Nigeria providing about $11.835 \mathrm{~kJ} /$ person/day (Osho, 2003). Gari is of poor quality and low protein content (less than $2.5 \%$ ) hence there is need for continual investigation into cheaper and better ways of enriching the product in order to improve its acceptability in terms of cost and nutritional quality. Soy-melon Gari is a response to this. Soy-melon gari is a product to which soy and melon protein flour had been added during the processing of gari.

Gari is still being packaged, transported and stored in woven sacks with attendant fluctuations in climatic conditions and sometimes it is being sold in the market in bowls with exposed surfaces thus increasing its susceptibility to environmental contaminations (Ogiehor \& Ikenebomeh, 2006). The price of gari is subjected to seasonal variation being low in the raining season and high in the dry season, with attendant great hardship to poor members of the society since gari is no more the food of the commoner. Gari is becoming more important in export trade hence it is necessary to monitor what happens to gari during long term storage. With the continued interest in the enrichment of gari with local protein sources from oilseeds, it is necessary to study the methods and the effects of the enrichment on storage stability.

In the purchase of fresh samples of gari in the market, appearance which encompasses the colour, finess or particle size seems to be the most striking sensory property attracting a consumer of gari in the market, after this is the taste for some people who love their gari to be sour. However, in the case of soy-melon gari, because of the oily nature of its supplement, flavour is very important in assessing the sensory quality of soy-melon gari that 
has undergone storage. The objectives of this study are therefore to produce soy-melon residue flours from combination of soy and melon flours; subject the fresh samples of soy-melon gari to sensory evaluation to determine the best supplement that had been used for the enrichment and evaluate their sensory (flavour) qualities during storage.

\section{Materials and Methods}

\subsection{Source of Materials}

Cassava roots harvested and used on the same day were obtained from the research farm of the Federal University of Technology, Akure, Ondo State, Nigeria. Soybean and melon seeds used to produce the protein supplements were purchased from the Oja Oba market, in Akure, Ondo State, Nigeria. They were sorted, cleaned, packed and kept under refrigeration until use.

\subsection{Sample Preparation}

Soy-melon gari was produced according to the methods of Banjoh Ikenebomeh (1996) and Adeyemi and Balogh (1985). The Cassava tubers were peeled manually with a sharp knife, washed and grated in a locally fabricated mechanical grater. The grater was made of a flat galvanized sheet punctured with holes with a big nail with opening of $0.75 \mathrm{~cm}$ diameter and fixed round a drum-like plank. This was connected through a belt to a $7 \mathrm{hp}$ driving motor. The washed cassava tuber was held by hand and ran over the rotating drum with extreme care that fingered and palm are not bruised (Agunbiade, 2001). They were then packed into Hessian sack and allowed to ferment for 72 hours after which they were pressed in a mechanical press (Addis Engineering Nig. Ltd, Nigeria) to dewater the mash. The dewatered wet cassava cake was pulverized with hands and sifted on a locally made raffia sieve of mesh $(0.3 \mathrm{~cm} \times 0.3 \mathrm{~cm})$ mounted on a rectangular wooden frame $40 \mathrm{~cm}^{2}$ to remove the fibers. The sifted cassava meal obtained was enriched with full fat soy-melon using $10 \%$ enrichment level and taking into consideration the water content of the mash of $65 \%$ (Akingbala et al., 1993). The white and fluffy cassava meal was then introduced into a wide iron pan (garifier) supported and being heated over wood fire. It was being continuously stirred using a self insulating manual baffle made of calabash from gourd. This operation fairly distributes the heat to prevent or limit dextrization of gari. The wet meal was being introduced into the garifier piecemeal, amidst continuous stirring, until a full manageable batch is subjectively determined as done. The time taken to get the batch roasted to dryness depends on the experience of the processor. From past studies, 1.5-2.2 $\mathrm{kg}$ of gari semolina was satisfactorily roasted over fire between 13-20 minutes, with high intensity of heat (Agunbiade, 2001). The toasted soy-melon gari was removed from the iron pot, spread over a large spread of clean surface of HDPE film and allowed to cool. The cooled gari samples were then packaged in HDPE film and kept under refrigerated storage until ready for further analysis. Part of the resulting product was reconstituted and subjected to sensory.

\subsection{Sensory Evaluation}

\subsubsection{Sensory Evaluation of Reconstituted Fresh Gari Granules ("eba”)}

The reconstituted "eba", a stiff dough from fresh soy-melon and control gari were evaluated in terms of colour (appearance), taste, aroma, texture and overall acceptability. The parameters were evaluated by a trained panel of judges who were already used to gari consumption. The samples were scored on a 9 point hedonic scale ranging from 9 = liked extremely to 1 = disliked extremely (IFT, 1981; Larmond, 1982). The "eba" was prepared by reconstituting gari with boiling water in ratio $1: 3(\mathrm{w} / \mathrm{v})$ by pouring the gari into already boiled water, in a small bowl, with hand, little by little until the gari imbibe all the water. The bowl was covered with a lid to allow proper gelatinization for about 3 minutes, after which it was mixed well with a spoon to form uniform stiff dough (Oluwamukomi et al., 2005; Edwards et al., 1977). A 20 member panel consisting of both staff and students was used. The samples were coded with 3-digit random numbers. The order of presentation of the samples was randomized for each panelist. The panelists were served the dough and asked to observe the appearance to check for the colour whether it was light or dark, yellow or brown; finger feel to check for texture whether it was gritty or smooth, soft or thick; taste test to score the taste whether it was sour or not. They were also to sniff test to detect any objectionable odour, beany flavour or any off flavour. The overall acceptability was evaluated by eating a small ball of dough which had been dipped into a stew prepared with water, beef, tomatoes, onion, salt, red hot pepper and seasoning blend (Collins \& Temalilwa, 1981). Results obtained from the mean panel scores were subjected to analysis of variance (ANOVA). Means were separated using The Duncan's New Multiple Range Test (Steel et al., 1997). 


\subsubsection{Sensory Evaluation of Stored Soy-Melon and Control Gari}

Soy-melon enriched and control gari samples stored at 20,30 and $40^{\circ} \mathrm{C}$ were assessed for quality on a nine-point hedonic scale (IFT, 1981; Larmond, 1982) by twenty experienced judges who were used to the consumption of gari. Fifty-gram samples packed and stored in pouches were evaluated for off-flavour at every four weeks interval covering a period of slightly over 32 weeks by a sensory panel consisting of 20 members (Labuza \& Schmidl, 1988). Most panel members were undergraduate students and staff members of the Department of Food Science and Technology who were used to the sensory evaluation of gari. Therefore, they were reasonably well trained for sensory tests. They were requested to evaluate differences in overall sensory quality between the control and other samples. A coded control sample was included in each set of samples evaluated in order to check the reliability of each panel member and to provide a base score for each sample series (Kluter et al., 1996). A nine-point flavor scoring system was used for the evaluation. No, slight, moderate, large and extreme differences were represented by scores of $1,3,5,7$ and 9, respectively. According to my preliminary test, scores less than 5 represent overall quality comparatively worse than the control gari and scores greater than 5 definitely better than the control gari sample (Hayakawa et al., 1978). In addition to this analysis, all data on the flavor difference scores for each product were subjected to regression analysis. Samples held at $10^{\circ} \mathrm{C}$ were used as the controls in all flavor evaluations, since the quality of these samples should be almost constant throughout my study. Therefore, the flavor difference scores included the influence of storage time, temperature, packaging, BHT and enrichment, as well as variations due to power outages (Hayakawa et al., 1978). In order to eliminate the influence of power outages, data on the flavor scores of the controls were excluded from the regression analysis since the relative differences in the scores of all other samples should represent the influence of storage time and temperatures. The shelf lives of soy-melon and control gari were calculated from the mean panel scores for flavour respectively by simple linear regression equation (Hayakawa et al., 1978; Steel et al., 1997).

\subsection{Statistical Analysis}

Means and standard errors of the mean (SEM) of replicate scores were determined and subjected to analysis of variance (ANOVA). Means were separated using The Duncan's New Multiple Range Test (Steel et al., 1997). Relevant data were subjected to correlation and regression analyses. All analyses were carried out using Microsoft Excel Office (2003) and the Statistical Package for Social Statistics (SPSS version 12).

\section{Results and Discussion}

\subsection{Sensory Evaluation of Reconstituted Gari Enriched and Control Gari Samples}

From Tables 1, 2 and 3, it was shown that there was a significant difference between the 'eba' made from un-enriched and the enriched gari granules $(\mathrm{P} \leq 0.05)$. It has been observed that consumers judge gari quality based on its appearance colour and with particle size being the most attributes of quality (Blanchard, 1994). In the case of reconstituted gari ("eba"), finger feel and mouth feel (texture) are its most important eating qualities (Oluwamukomi et al., 2005). Eba should not be too soft or too sticky when tested by the finger and it should have a smooth feel in the mouth and be easily swallowed usually without chewing when put in the mouth as a round ball (Almazan, 1992). The sensory characteristics of enriched eba depend on the level of enrichment. On the basis of appearance, there was no significant difference in the colour of the soy-melon gari from different supplement. Toasting gari with any of the supplements will not impart any significant different effect from each other. However, the control sample with no supplement was significantly scored better than the enriched gari ( $\mathrm{P}$ $<0.05$ ). This is slightly different from the findings of Oluwamukomi et al. (2005), and Collins and Temalilwa (1981) that there was no different in the control and enriched gari with soy flour alone up to $10 \%$ enrichment and Banjoh and Ikenebomeh (1996) up to 15\% enrichment level. Enrichment reduced the mean panel scores showing that they were less accepted than the control sample. The panel scores was reduced from 7.9 in the control sample to a range of 3.47 to 5.37 with sample enriched with full-fat supplement before fermentation being the least scored (3.47) and the sample toasted together with defatted flour being the highest scored (6.6).

On the basis of texture which was to show how soft or thick, smooth or gritty the sample was, it was shown that the control was the best, closely followed by the gari enriched with full fat during toasting. The worst sample was the sample enriched before fermentation. On the basis of flavour or off-flavour, the control sample was the best followed by samples enriched with defatted and milk residues after fermentation with scores of 7 for the two. On the basis of overall acceptability, gari enriched with full fat soy melon after fermentation gave the best overall accepted product after the control sample. 
Table 1. Mean panel scores for the reconstituted soy-melon and control gari

\begin{tabular}{lllllll}
\hline Sample & & Appearance & Texture & Taste & Flavour & Overall acceptability \\
\hline DEFATTED & BF & $4.9 \pm 0.31$ & $6.0 \pm 0.21$ & $6.2 \pm 0.34$ & $5.8 \pm 0.20$ & $6.0 \pm 0.21$ \\
& DT & $6.4 \pm 0.37$ & $4.0 \pm 0.21$ & $4.1 \pm 0.023$ & $7.0 \pm 0.21$ & $8.6 \pm 0.21$ \\
& AT & $4.8 \pm 0.29$ & $3.0 \pm 0.21$ & $3.42 \pm 0.023$ & $3.8 \pm 0.20$ & $4.8 \pm 0.20$ \\
\hline FULL FAT & BF & $2.6 \pm 0.27$ & $3.6 \pm 0.25$ & $5.2 \pm 0.031$ & $5.1 \pm 0.18$ & $4.0 \pm 0.21$ \\
& DT & $6.3 \pm 0.27$ & $7.2 \pm 0.18$ & $7.4 \pm 0.024$ & $5.6 \pm 0.22$ & $8.2 \pm 0.20$ \\
& AT & $5.7 \pm 0.34$ & $3.2 \pm 0.33$ & $5.1 \pm 0.022$ & $4.0 \pm 0.21$ & $5.0 \pm 0.21$ \\
\hline MILK & BF & $2.9 \pm 0.18$ & $2.9 \pm 0.23$ & $5.3 \pm 0.031$ & $5.0 \pm 0.21$ & $2.9 \pm 0.23$ \\
RESIDUE & DT & $6.8 \pm 0.13$ & $6.9 \pm 0.18$ & $6.5 \pm 0.013$ & $7.0 \pm 0.21$ & $7.0 \pm 0.26$ \\
& AT & $3.6 \pm 0.22$ & $4.1 \pm 0.18$ & 4.120 .014 & $4.0 \pm 0.21$ & $4.9 \pm 0.18$ \\
\hline CONTROL & & $7.9 \pm 0.18$ & $8.8 \pm 0.13$ & $8.8 \pm 0.13$ & $8.8 \pm 0.13$ & $8.9 \pm 0.10$ \\
\hline
\end{tabular}

Values are means of 20 replicate panel scores;

\pm Standar errors of mean;

Key: $\mathrm{BF}=$ Enrichment before fermentation (wet mix method);

DT = Enrichment during toasting (soak mix method);

$\mathrm{AT}=$ Enrichment after toasting (dry mix method).

Table 2. Effect of type of supplement on the mean panel scores of reconstituted soy-melon and control gari

\begin{tabular}{llllll}
\hline Type of Supplement & Appearance & Texture & Taste & Flavour & Overall acceptability \\
\hline DEFATTED & $5.37^{\mathrm{b}}$ & $4.33^{\mathrm{b}}$ & $4.12^{\mathrm{c}}$ & $5.53^{\mathrm{b}}$ & $6.27^{\mathrm{b}}$ \\
FULLFAT & $4.97^{\mathrm{b}}$ & $4.77^{\mathrm{b}}$ & $4.34 \mathrm{c}$ & $4.90^{\mathrm{b}}$ & $5.73^{\mathrm{bc}}$ \\
MILK RESIDUE & $4.43^{\mathrm{b}}$ & $4.63^{\mathrm{b}}$ & $5.31^{\mathrm{b}}$ & $5.33^{\mathrm{b}}$ & $4.93^{\mathrm{c}}$ \\
CONTROL & $7.90^{\mathrm{a}}$ & $8.80^{\mathrm{a}}$ & $8.80^{\mathrm{a}}$ & $8.80^{\mathrm{a}}$ & $9.90^{\mathrm{a}}$ \\
\hline
\end{tabular}

Values are means of 20 replicate panel scores;

a,b,c * Means followed by different letters in a column are significantly different $(\mathrm{P} \leq 0.05)$;

Key: $\mathrm{BF}=$ Enrichment before fermentation (wet mix method);

DT $=$ Enrichment during toasting (soak mix method);

AT= Enrichment after toasting (dry mix method).

Table 3. Effect of point of inclusion of supplement on the mean panel scores of reconstituted soy-melon and control gari

\begin{tabular}{llllll}
\hline Stage of Enrichment & Appearance & Texture & Taste & Flavour & Overall acceptability \\
\hline BF & $3.47^{\mathrm{d}}$ & $4.37^{\mathrm{b}}$ & $5.3 \mathrm{~b}^{\mathrm{c}}$ & $5.30^{\mathrm{c}}$ & $4.30^{\mathrm{c}}$ \\
DT & $6.60^{\mathrm{b}}$ & $5.93^{\mathrm{ab}}$ & $6.71^{\mathrm{b}}$ & $6.53^{\mathrm{b}}$ & $7.73^{\mathrm{b}}$ \\
AT & $4.70^{\mathrm{c}}$ & $3.43^{\mathrm{c}}$ & $3.51^{\mathrm{c}}$ & $3.93^{\mathrm{d}}$ & $4.90^{\mathrm{c}}$ \\
CONTROL & $7.90^{\mathrm{a}}$ & $8.80^{\mathrm{a}}$ & $8.88^{\mathrm{a}}$ & $8.80^{\mathrm{a}}$ & $9.90^{\mathrm{a}}$ \\
\hline
\end{tabular}

Values are means of 20 replicate panel scores;

a,b,c $*$ Means followed by different letters in a column are significantly different $(\mathrm{P} \leq 0.05)$;

Key: $\mathrm{BF}=$ Enrichment before fermentation (wet mix method);

DT = Enrichment during toasting (soak mix method);

$\mathrm{AT}=$ Enrichment after toasting (dry mix method). 


\subsection{Effect of Type and Point of Inclusion of Supplement on the Sensory Properties of Reconstituted Soy Melon Gari ("eba")}

From Table 2, it could be observed that there was no significant effect of type of supplement on the appearance of soy-melon gari when reconstituted into eba $(\mathrm{P}>0.05)$ even though the scores were different, they were not statistically significant. Type of supplement used did not influence the appearance or colour of the eba. However the control sample was significantly different from the rest of the samples. All the samples were equally accepted based on the type of supplement used. However, based on the effect of the point of inclusion or stage of enrichment, Table 3 shows that the sample enriched before fermentation had a very low score (3.47) which was rejected by the panelist as being too dark. This darkness in colour was also observed in a preliminary study by Oluwamukomi et al. (2005) which means that the darkness might have been as a result of the processing rather than the supplement. In the study carried out by Oluwamukomi et al. (2005), soy flour alone was used as the supplement and the colour was also darkened. This showed that it could have been as a result of product of fermentation of the soy-melon flour together with the cassava wet meal which further went through toasting. Products of fermentation formed could have further led to Maillard reaction or lipid oxidation with attendant darkening of the colour (Ihekoronye \& Ngoddy, 1985). The sample enriched after toasting also had a low colour score but was not out rightly rejected as the sample enriched before fermentation. The colour was also darkened especially in the samples enriched with full fat soy-melon supplement.

Similarly the texture of the eba from the soy-melon gari was significantly different from the control. When the reconstituted eba was finger tested the texture of the enriched gari were softer than the control especially samples enriched before fermentation. The texture was a bit more fluffy and non cohesive. However at the $10 \%$ enrichment level it was still acceptable as the control sample. The flavour of soy-melon gari enriched at the different stages were significantly different from the flavour of the control sample, but the beany flavour were not very pronounced at this enrichment level and were not significantly different from each other. In terms of the taste, the enriched samples were less sour than the control sample, but the sample toasted together with the supplement, still tasted as the control.

On the basis of overall acceptability, the control sample was found to be the best followed by the sample enriched with defatted supplement, closely followed by sample enriched with full fat and milk residue in that order. Samples enriched before fermentation were softer and brownish in color while that enriched after toasting was more fluffy and less visco- elastic than the control sample. This was consistent with previous finding of earlier workers (Oluwamukomi et al., 2005, Banjoh \& Ikenebomeh, 1996; Oshodi, 1985). Oshodi (1985) observed that "eba" from $25 \%$ soy and $25 \%$ cooked melon samples were rejected as being too soft and lack cohesiveness, but when he used uncooked melon and the combined product from soybean and uncooked melon; the "eba" samples were accepted, which he attributed to the uncooked melon. He suggested the use of binders to improve cohesiveness at that level of enrichment so as to cut down on the amount of gari to be used in making "eba". In terms of the taste and aroma, sample from the soak-mix method was slightly sourer than the other enriched products but not as much as that of the control sample.

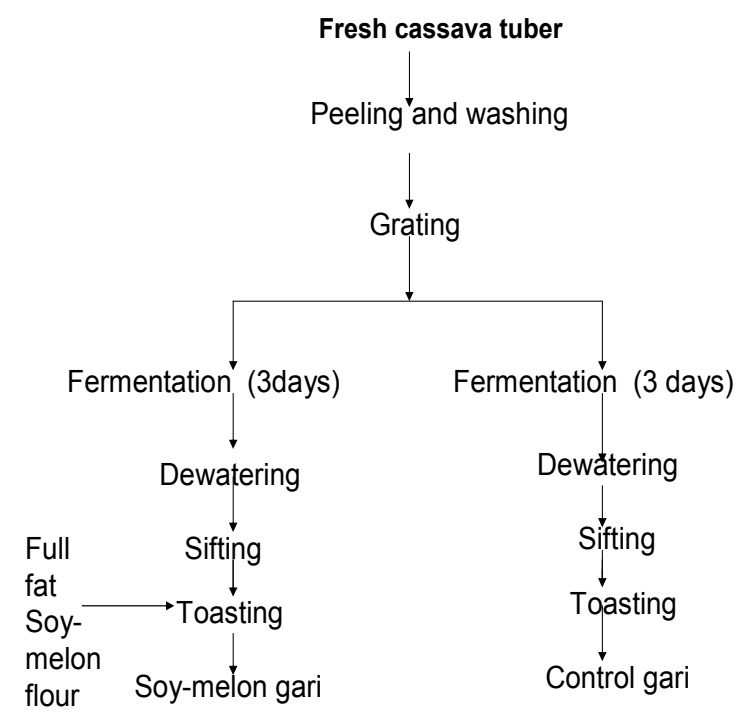

Figure 1. Flow chart of the processing techniques for control (un-enriched) and soy-melon enriched gari samples 


\subsection{Effect of Enrichment on the Flavour Score of Soy-Melon Gari Semolina}

Figure 2 shows the effect of enrichment on the flavor scores of soy-melon gari semolina during storage. Enrichment had a significant effect on the flavour score of soy-melon gari over storage at accelerated temperature of $40^{\circ} \mathrm{C}(\mathrm{P} \leq 0.05)$. The mean panel flavour score decreased with time and the off flavour became strong after 18 weeks of storage when the mean panel flavour score for samples kept in Woven sack and HDPE at $40^{\circ} \mathrm{C}$ reached mean critical score of 5 at 18 and 22 days respectively. The control (un-enriched) product did not reach the mean critical score throughout the 32 weeks period of storage. The faster decrease in flavour of the soy-melon gari than that of the control gari sample might have been due to the effect of oil $(23.3 \%)$ in the soy-melon supplement. This might have been due to the oxidation of the unsaturated fatty acid of the lipid component in the soy-melon supplement resulting in off flavour compounds such as hexanal with grassy flavour (Salunkhe et al., 1992). The off flavour could be as a result of oxidation or by lipoxygenase catalysis. The presence of a significant amount of polyunsaturated fatty acids, linoleic and linolenic acids and the enzyme lipoxygenase are responsible for the production of off-flavour and off aroma compounds at various stages of processing (Iwe, 2003). Iwe (2000) also observed that the levels of tri-hydroxyl acid in ground soybean and defatted meal increased with storage.

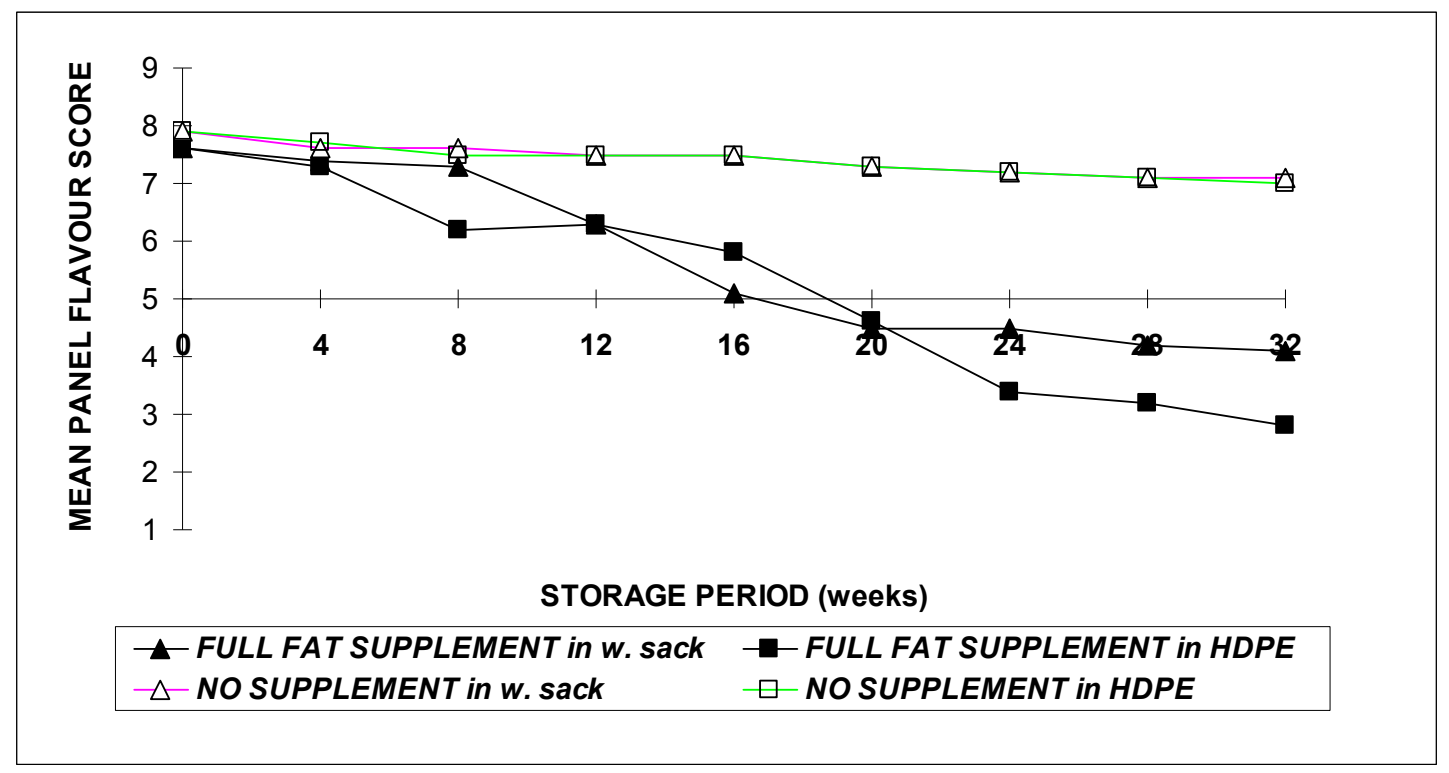

Figure 2. Effect of soy-melon enrichment on the mean flavour scores of soy-melon gari semolina under storage at $40^{\circ} \mathrm{C}$

\subsection{Effect of Enrichment on the Colour Scores of Soy-Melon Gari Semolina}

The effect of enrichment on the mean colour score of soy-melon gari semolina under storage are presented in Figure 3. Enrichment had a significant effect $(\mathrm{p} \leq 0.05)$ on the colour score of soy-melon gari under storage at accelerated temperature of $40^{\circ} \mathrm{C}$. The mean panel colour score decreased faster with time for enriched gari than the un-enriched gari. The mean colour score was reached at 18 and 22 weeks for soy-melon enriched gari packaged in woven sack and HDPE respectively; while the control (un-enriched) gari did not reach the critical mean colour score (5) throughout the study and did not change in colour. The darkening and browning in colour might have been due to the effect of enrichment with soy-melon supplement of high lipid content. It might have been due to the oxidation of the unsaturated fatty acid of the lipid component of the full fat soy-melon supplement used for the enrichment. 


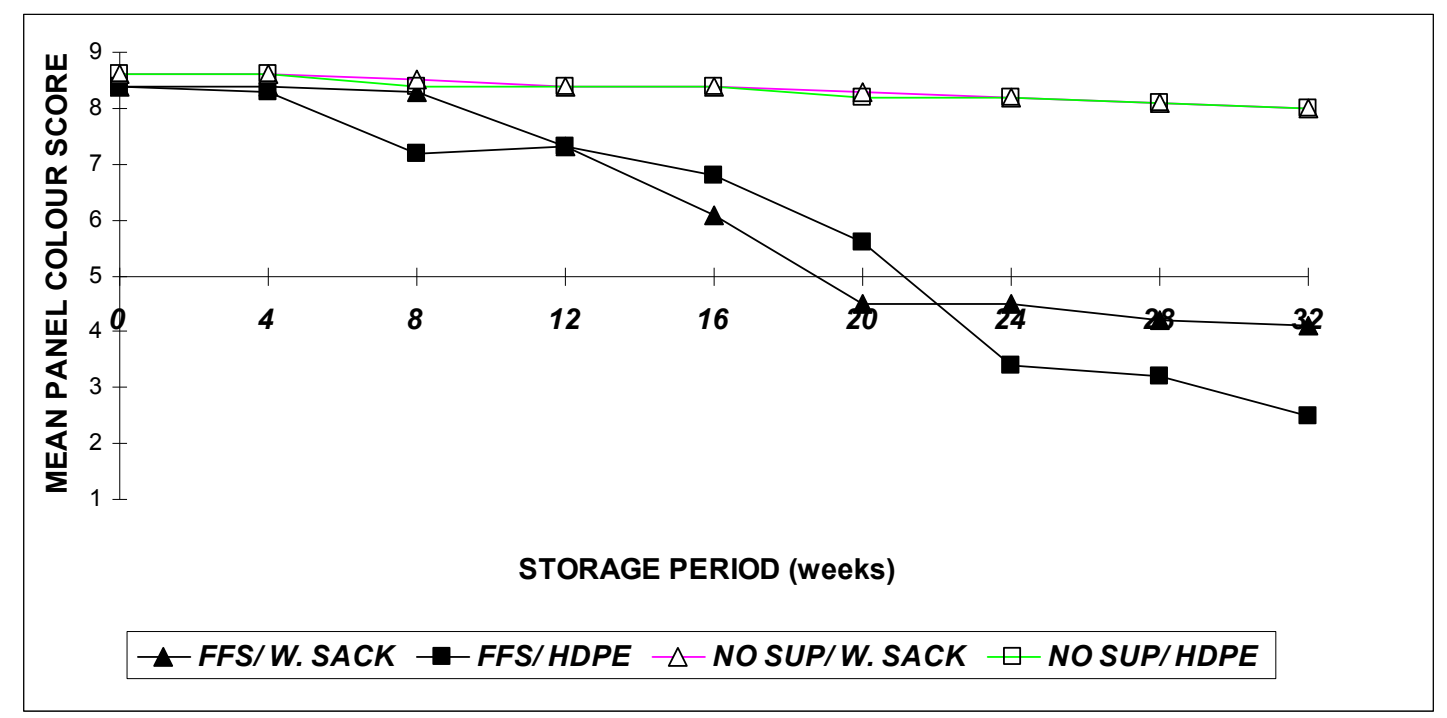

Figure 3. Effect of soy-melon enrichment on the mean colour score of soy-melon gari under storage at $40^{\circ} \mathrm{C}$

\section{Conclusions}

This study was carried out in order to investigate the best point of inclusion of soy-melon protein supplement in the manufacture of 'gari' and also to establishes the factors affecting its shelf life and predicts the shelf life of gari enriched with full fat soy-melon supplement. On the basis of the sensory evaluation of gari and its reconstituted product, enrichment did not significantly influence the appearance or colour of the eba. However the control sample was significantly different from the rest of the samples. All the samples were equally accepted. On the effect of the point of inclusion (or stage of enrichment), the sample enriched before fermentation (wet mix) had a very low score and was rejected by the panelist as being too dark. The sample enriched after toasting (dry mix) also had a low colour score but was not out rightly rejected as the sample enriched before fermentation. The texture of the eba from the soy-melon gari was softer than the control, especially samples enriched before fermentation. The flavour of soy-melon gari enriched at the different stages was significantly different from the flavour of the control sample, but the beany flavour was not very pronounced at this enrichment level $(15 \% \mathrm{w} / \mathrm{w} \mathrm{db})$ and were not significantly different from each other. In terms of the taste, the enriched samples were less sour than the control sample. Generally, the control gari was rated to be the best followed closely by the sample enriched with defatted supplement, followed by the sample enriched with full fat soy-melon meal and then the sample enriched with the milk residue in that order. Samples enriched before fermentation (soak mix) were softer and brownish in color while those enriched after toasting (dry mix) were more fluffy and less visco- elastic than the control sample. The sample enriched before toasting and toasted together with cassava meal was scored to be the best point at which enrichment should be carried out.

This leads to the conclusion that the best soy-melon gari is the one produced by supplementing cassava meal with defatted supplement just before toasting commences and toasted together with the meal. However because of the attendant cost of de-fatting soy-melon meal and the logistic problems likely to be involved any of the other two methods of enriching with full fat or milk residue meal supplements could be practiced at cottage level. The milk residue is recommended if it could be done hygienically but the problem is the high moisture content which may be difficult to remove at the cottage level. Enrichment also reduced drastically the shelf life when soy-melon gari was stored at elevated temperature of $40^{\circ} \mathrm{C}$. The oil in the full fat soy and melon meal must have been responsible for this. To prevent this, enrichment with defatted supplement should be encouraged wherever it is possible in other to increase the shelf life. Shelf life prediction by the sensory evaluation methods correlated positively with those of half life and shelf life plot methods used in this study. What this means is that any of these methods that correlated positively with sensory evaluation, could be used to predict the shelf life of soy-melon gari successfully.

\section{References}

Adeyemi, I. A., \& Balogh, E. (1985). Biotechnology in food processing-Prospects in Nigeria. Nig. Food Journal, $3(1,2,3), 33-39$. 
Agunbiade, S. O. (2001). Effect of fermentation period on the physicochemical properties of gari. Bioscience Research Communications, 13(1), 65-71.

Akingbala, J. O., Oguntimehin, G. B., \& Bolade, M. K. (1993). Effect of unit operations of production on the cyanide content and acceptability of gari. J. Food Processing and Preservation, 17, 337-380. http://dx.doi.org/10.1111/j.1745-4549.1993.tb00736.x

Almazan, (1992). Influence of cassava variety and storage on gari quality. Trop. Agric. (Trinidad), 69(4), 386-390.

Banjoh, N. O., \& Ikenebomeh, M. J. (1996). Comparison of 3 methods for the preparation of Soy gari from cassava and soybean mash. J. Food Science \& Tech., 33(4), 440-442.

Blanchard, A. F. J., Daniyan, M. T., Poulter, N., \& Taylor, .A. J. (1994). Quality of Cassava in Sierra Leone. J. Science. Agric., 64, 426-432.

Collins, J. L., \& Temalilwa, C. R (1981). Cassava (Manihot esculenta Crantz) flour fortification with soy flour. J. Food Sci., 46, 1025-1028. http://dx.doi.org/10.1111/j.1365-2621.1981.tb02984.x

Edwards, C. C., Onyekwere, O. O., \& Akinrele, I. A. (1977). Preliminary studies on protein enrichment of gari with peanut grits: 1. Formulation, characterization and organoleptic evaluation. Proceedings of Nig. Inst. Food Sci. and Tech., 1, 95-102.

Hayakawa, K., Matas, J., \& Hwang, M. P. (1978). Moisture sorption isotherm of coffee products. J. Food Sci., 43, 1026-1028. http://dx.doi.org/10.1111/j.1365-2621.1978.tb02479.x

IFT. (1981). Sensory evaluation guide for testing food and beverage products by the sensory. Evaluation division of the Institute of Food Technologists. Food Technol., pp. 50-59.

Ihekoronye, A. I., \& Ngoddy, P. O. (1985). Browning reaction. In Integrated Food Science and Technology for the Tropics (pp. 224-229). London: Macmillan Education Ltd.

Iwe, M. O. (2003). Soy Chemistry. In The Science and Technology of Soybean, Chemistry, nutrition, processing and utilization (pp. 27-56). Rojoint Communication Services Ltd.

Iwe, M. O. (2000). Effects of extrusion cooking on some functional properties of soy-sweet potato mixtures. Lebensm.-Wiss. U-Technol., 25, 569-573.

Kluter, R. A., Nattress, D. T., Dunne, C. P., \& Popper, R. D. (1996). Shelf Life Evaluation of Bartlett Pears in Retort Pouches. J. Food Science, 61(6), 1297-1302. http://dx.doi.org/10.1111/j.1365-2621.1996.tb10982.x

Kordylas, J. M. (1990). Processing and preservation of tropical and sub-tropical foods. London and Basingstoke: Macmillan.

Labuza, T. P., \& Schmidl, M. K. (1985). Accelerated shelf life testing of foods. Food Technol., 39(9), 57-62.

Lancaster, P. A. (1982). Traditional cassava based foods. In J. S. Igran, M. Y. Lim, \& D. G. Coursey (Eds.), Survey of processing techniques. Economic Botany, 36, 12-45. http://dx.doi.org/10.1007/BF02858697

Larmond, E. (1977). Methods of sensory testing. In Laboratory methods for sensory evaluation of foods. Ottawa: Canadian Dept of Agric.

Ogiehor, I. S., \& Ikenebomeh, M. J. (2006). The effects of different packaging materials on the shelf stability of garri. African Journal of Biotechnology, 5(23), 2412-2416.

Oluwamukomi, M. O., Adeyemi, I. A., \& Oluwalana, I. B. (2005). Effects of soybean enrichment on the physicochemical and sensory properties of gari. Applied Tropical Agriculture, 10, 44-49.

Osho, S. M. (2003). The processing and acceptability of a fortified cassava-based product (gari) with soybean. Nutrition \& Food Science, 33(6), 278-283. http://dx.doi.org/10.1108/00346650310507118

Oshodi, A. A (1985). Protein enrichment of foods that are protein deficient: Fortification of gari with soybean and melon. Nig. J. Applied Science, 3(2), 15-22.

Salunkhe, D. K., Chavan, J. C., Adsule, R. N., \& Kadam, S. S. (1992). World oilseeds, Chemistry, Technology and Utilization. New York: Van Nostrand Reinhold.

Steel, R., Torrie, J., \& Dickey, D. (1997). Principles and Procedures of Statistics: A Biometrical Approach (3rd ed.). New York, USA: McGraw Hill Book Co. 